\title{
Diagnosis of visceral pleural invasion using confocal laser endomicroscopy during lung cancer surgery
}

\author{
Toru Sawada ${ }^{1}$, Hiromitsu Takizawa ${ }^{1}$, Mariko Aoyama ${ }^{1}$, Naoya Kawakita ${ }^{1}$, Naoki Miyamoto ${ }^{1}$, \\ Shinichi Sakamoto ${ }^{1}$, Mika Takashima ${ }^{1}$, Daisuke Matsumoto ${ }^{1}$, Hiroaki Toba ${ }^{1}$, Yukikiyo Kawakami ${ }^{1}$, \\ Mitsuteru Yoshida ${ }^{1}$, Kazuya Kondo ${ }^{2}$, Akira Tangoku ${ }^{1}$
}

${ }^{1}$ Department of Thoracic, Endocrine Surgery and Oncology, Tokushima University Graduate School of Biomedical Sciences, Kuramotocho, Tokushima, Japan; ${ }^{2}$ Department of Oncological Medical Services, Tokushima University Graduate School of Biomedical Sciences, Kuramotocho, Tokushima, Japan

Contributions: (I) Conception and design: H Takizawa; (II) Administrative support: H Takizawa; (III) Provision of study materials or patients: T Sawada, H Takizawa, H Toba, M Yoshida, K Kondo; (IV) Collection and assembly of data: T Sawada, H Takizawa, M Aoyama, N Kawakita, N Miyamoto, S Sakamoto, M Takashima, D Matsumoto; (V) Data analysis and interpretation: T Sawada, H Takizawa, M Aoyama, N Kawakita; (VI) Manuscript writing: All authors; (VII) Final approval of manuscript: All authors.

Correspondence to: Hiromitsu Takizawa. Department of Thoracic, Endocrine Surgery and Oncology, Tokushima University Graduate School of Biomedical Sciences, 3-18-15, Kuramotocho, Tokushima 770-8503, Japan. Email: takizawa@tokushima-u.ac.jp.

Background: Visceral pleural invasion (VPI) in lung cancer is a significant prognostic factor; however, it is difficult to diagnose preoperatively or intraoperatively. In this study, we examined the possibility of intraoperative diagnosis of VPI using confocal laser endomicroscopy (CLE).

Methods: Among patients with primary lung cancer who underwent surgery between April 2018 and August 2019, those in whom the tumor was in contact with the pleura on chest computed tomography and whose pleural changes were intraoperatively confirmed were enrolled in this study. In the 35 patients who underwent lung resection (6 cases with visceral pleural infiltration), the area where pleural change was noted was observed and a short video was recorded using CLE. Based on the video images, three evaluators determined the defect ratio $(0 \%, 25 \%, 50 \%, 75 \%$, and $100 \%)$ of the autofluorescence-positive structure. The area under the receiver operating characteristic curve was used to evaluate the diagnostic performance for VPI. In 15 cases ( 3 cases with VPI), a validation study was performed for intraoperative VPI according to the cutoff value of the defect ratio of the autofluorescence-positive structure.

Results: The areas under the receiver operating characteristic curve for the defect ratio of the autofluorescence-positive structure were $0.86-0.91$ for the three readers. Using defect ratio of autofluorescence-positive structure cutoff of $\geq 50 \%$ as predictor of VPI, the sensitivity, specificity, positive predictive value, negative predictive value, and accuracy were $83.3-100.0 \%, 57.7-73.1 \%, 35.3-41.7 \%$, $95.0-100.0 \%$, and $75.0-78.1 \%$, respectively, for the three readers. In the validation study, the sensitivity was $100 \%$, the specificity was $83.3 \%$, and the diagnostic accuracy rate was $86.7 \%$.

Conclusions: The diagnosis of VPI through CLE is simple, non-invasive, and has high diagnostic accuracy rates. This method may be applicable for determining surgical procedures.

Keywords: Lung cancer; visceral pleural invasion (VPI); confocal laser endomicroscopy (CLE)

Submitted Mar 03, 2021. Accepted for publication Jun 19, 2021.

doi: $10.21037 /$ jtd-21-137

View this article at: https://dx.doi.org/10.21037/jtd-21-137

(C) Journal of Thoracic Disease. All rights reserved. 


\section{Introduction}

Lung cancer is one of the leading causes of malignant tumor deaths worldwide (1). Accurate staging is crucial when deciding on lung cancer treatment. In 2017, the International Association for the Study of Lung Cancer revised lung cancer staging. The revision further subdivided the $\mathrm{T}$ factor into the TNM classification (2). Among them, visceral pleural invasion (VPI) is a significant prognostic factor that determines the $T$ factor. Even if the tumor is $\leq 3 \mathrm{~cm}$, if VPI is present, then the $\mathrm{T}$ factor will be $\mathrm{T} 2 \mathrm{a}$ and the staging at IB or higher.

In recent years, with the spread and higher performance of computed tomography (CT) devices, the probability of detecting small lung tumors have increased. In addition, the usefulness of sublobar resection for small lung tumors has been reported $(3,4)$. Lobectomy remains the standard surgery for lung cancer; however, depending on the results of the ongoing Phase 3 study (JCOG0802WJOG4607L) (5), segmental resection may be one of the standard surgical procedures for small lung tumors that are $2 \mathrm{~cm}$ or smaller.

The prognosis of patients with VPI is poor even in small lung tumors of $2 \mathrm{~cm}$ or smaller $(6,7)$. It has been reported that $9.7-13.8 \%$ of patients preoperatively diagnosed with cT1aN0M0 have VPI (8-10). Limited discussion on the exclusion of stage IB VPI cases from the indication of sublobar resection is due to the absence of a preoperative or intraoperative diagnostic method for VPI.

Confocal laser endomicroscopy (CLE) is a novel technology for imaging cells (11). Confocal technology was originally developed as a technology to be mounted on a microscope. This technology made it possible to not only visualize the tissue surface but also take deep images at high magnification. CLE is an application of this technology to endoscopes. While a normal endoscope can only magnify surfaces, CLE was developed to obtain observation images at the same level as the pathological image of a biological specimen. Although the confocal observation system unit is built into the tip of the endoscope, the confocal image can be observed in real time at the same time as the normal observation image without compromising the resolution of the confocal image.

The clinical application of CLE is not limited to the gastrointestinal region (12). Moreover, its applicability for malignant tumors and inflammatory diseases in the skin and oral cavity has been reported $(13,14)$. In the respiratory region, it has been observed that evaluation of the tracheobronchial mucosa by CLE in bronchoscopy is effective in diagnosing malignant neoplasms and inflammatory diseases (15). CLE emits 488-nm excitation light, and this technique makes it possible to obtain highquality images from endogenous or exogenous tissue fluorophores. As a result, it has been shown to visualize the elastin component in the basement membrane of lung tissue (16). Previously, we focused on the reduction or disappearance of autofluorescence in the pleura with lung cancer invasion. We reported the usefulness of pleural autofluorescence observation using a fluorescent endoscopy system for intraoperative diagnosis of VPI in lung cancer (17).

In this study, our focus has shifted to non-invasive, realtime image diagnosis in CLE. The purpose of this study was to examine the possibility of VPI diagnosis by observing the visceral pleural surface of the tumor site during surgery by touching it with a CLE probe.

We present the following article in accordance with the STARD reporting checklist (available at https://dx.doi. org/10.21037/jtd-21-137).

\section{Methods}

\section{Patients}

Patients who underwent thoracoscopic surgery for primary lung cancer at Tokushima University Hospital between April 2018 and August 2019 were enrolled. Patients whose tumors were in contact with the pleura based on preoperative CT and whose pleural changes were intraoperatively confirmed were included. Patients with intrapleural adhesions were excluded because this would hinder the identification of pleural changes. Written consent was obtained from each patient. Thirty-five cases in the first half of the study period were prospectively included in an ex vivo study to set the diagnostic criteria for VPI of lung cancer by CLE. In the latter half of the study period, 15 cases were prospectively included in the validation study (in vivo study) of the VPI diagnostic criteria by CLE. The study was conducted in accordance with the Declaration of Helsinki (as revised in 2013). The study was approved by the Ethics Review Board of the Tokushima University Hospital (approval number 3362), and informed consent was obtained from all the participants.

\section{Equipment}

CLE is based on the principle of illuminating tissue with the use of a low-power laser and then detecting the 

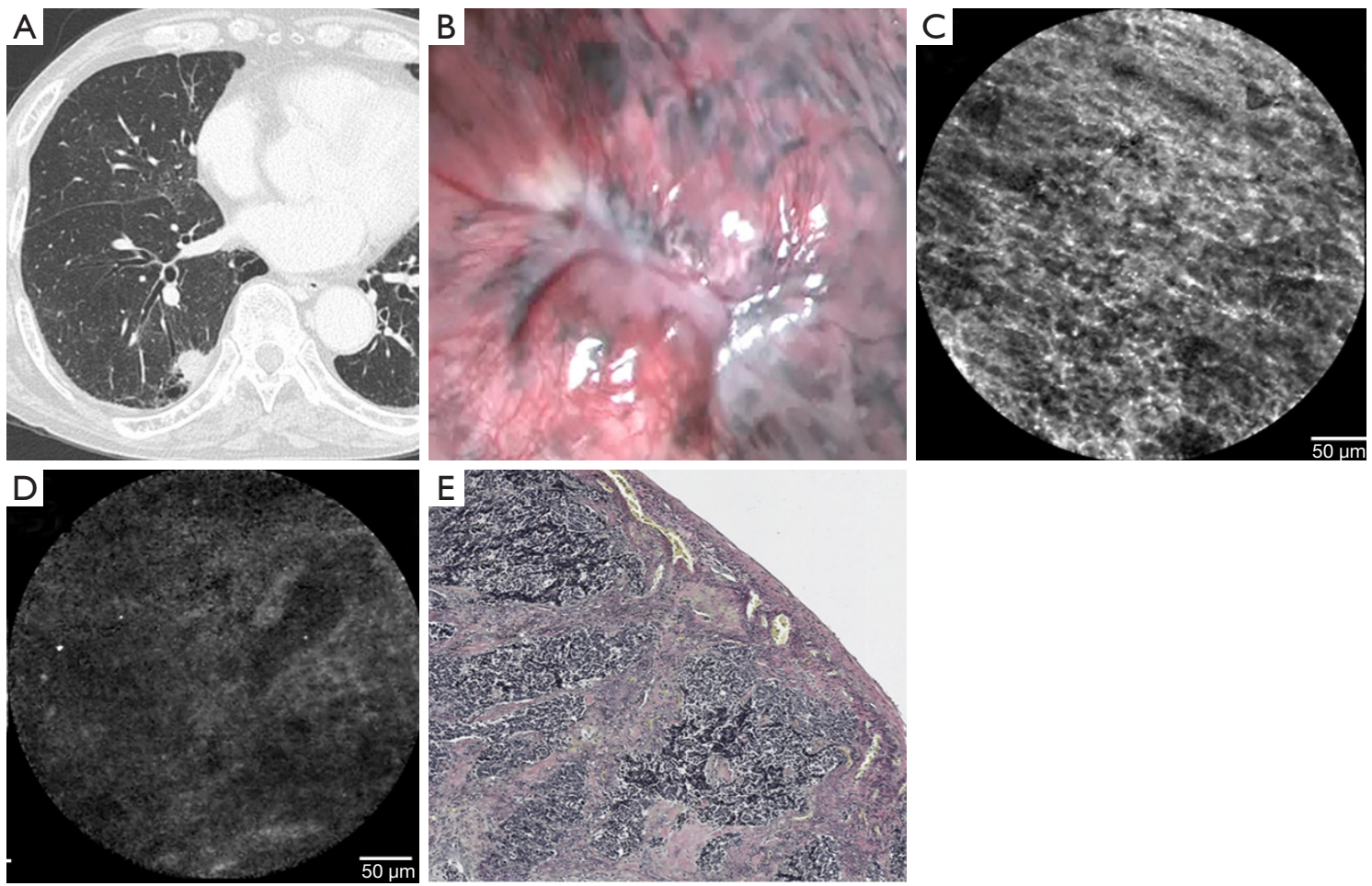

Figure 1 Cases without visceral pleural invasion (p10). (A) Chest CT shows a tumor in contact with the interlobar pleura; (B) macroscopic findings of the resected lung show depression and whitening in the visceral pleura at the tumor site; (C) CLE findings of the visceral pleura without tumor show a network structure; (D) CLE findings of the visceral pleura at the tumor site show an autofluorescence-positive structure; (E) Elastica van Gieson stain shows no tumor invasion in the visceral pleura, and the distance between the pleural surface and the external elastic membrane was short $(\times 25)$.

fluorescent light reflected from the tissue. The laser is focused at a specific depth, and only the light reflected back from the plane is refocused through a pinhole confocal aperture. Thus, scattered light from below and above the plane of interest is not detected, and the special resolution is enhanced. The examined area is scanned in the horizontal and vertical planes, and an image is reconstructed (15).

CLE was performed with the Cellvizio system (Mauna Kea Technologies, Paris, France) operating at a wavelength of $488 \mathrm{~nm}$. A 1.4-mm diameter confocal miniprobe (AlveoFlex; Mauna Kea Technologies) was used. The probe has a lateral resolution of $3.5 \mathrm{~mm}$, a field of view of $600 \times 500$ $\mu \mathrm{m}$, and a penetration depth of $0-50 \mu \mathrm{m}$.

\section{Case presentation and bypothesis}

Here, we present the difference in CLE findings based on the presence of VPI. Case 1 was a pathologic nonVPI (p10) lung adenocarcinoma. Preoperative chest
CT examination revealed a tumor in contact with the pleura between the upper and lower right lobes and the accompanying pleural indentation image (Figure 1A). Observation with a thoracoscope revealed a whitish change and pleural indentation at the tumor site (Figure 1B). A thoracoscopic right lower lobectomy was performed, and the CLE probe was brought into contact with the visceral pleura of the resected lung for observation. A regular pattern of autofluorescence-positive network structure was confirmed at the site of the normal pleura away from the tumor (Figure 1C). In contrast, the central part of the pleural change showed a rough wavy structure; however, the autofluorescence-positive structure did not disappear (Figure 1D). Pathologically, no VPI and no pleural thickening was observed (Figure 1E).

Case 2 involved (pl1) lung adenocarcinoma with pathologic VPI. Preoperative chest CT examination revealed a tumor in contact with the pleura (Figure $2 A$ ). Observation with a thoracoscope revealed a whitish change 

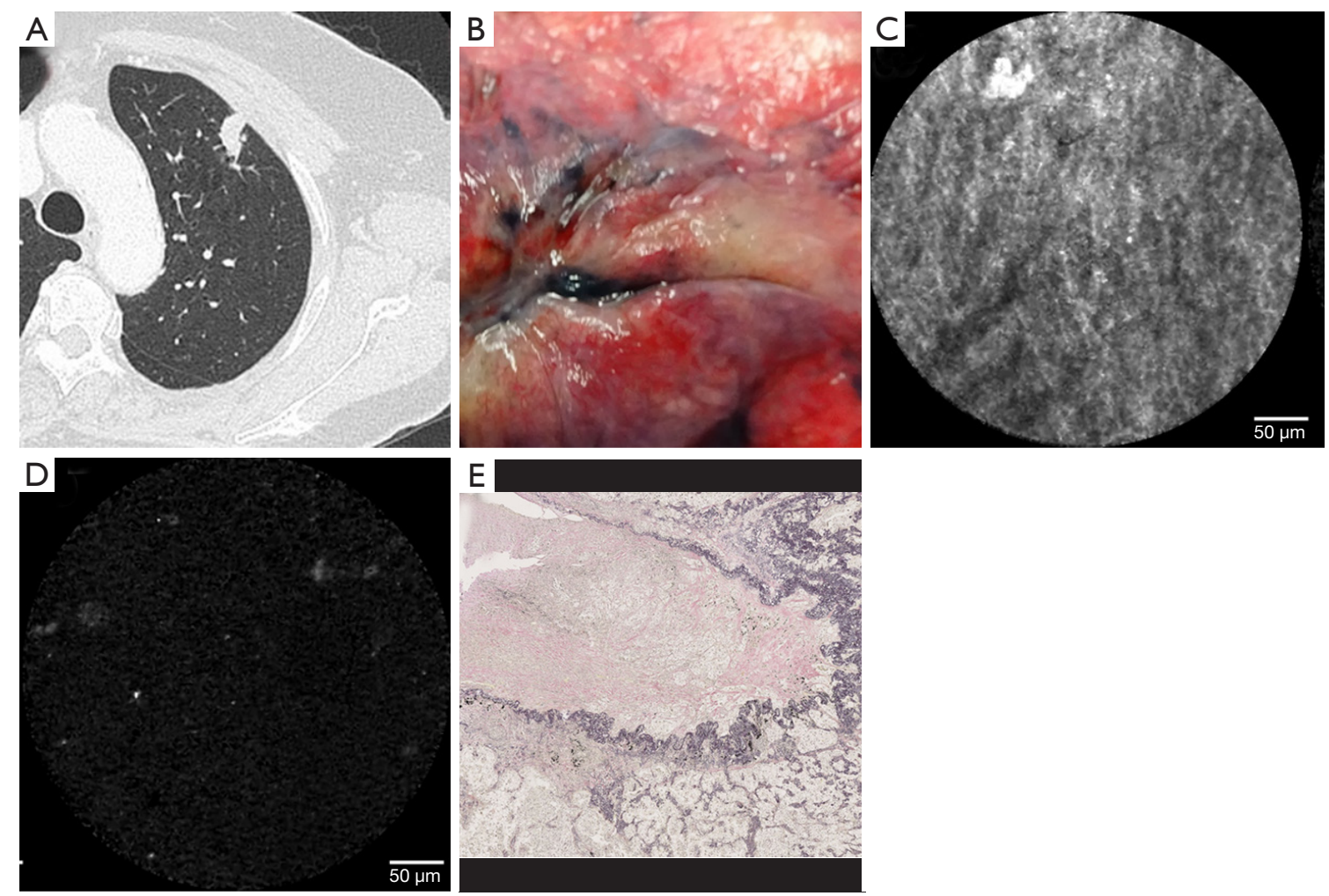

Figure 2 Case of visceral pleural invasion (pl1). (A) Chest CT shows a tumor in contact with the parietal pleura; (B) Macroscopic findings of the resected lung show depression and whitening in the visceral pleura at the tumor site; (C) CLE findings of the visceral pleura without tumor show a network structure; (D) CLE findings of the visceral pleura at the tumor site show a lack of autofluorescence-positive structure; (E) Elastica van Gieson stain shows visceral pleural invasion, and a thick layer of collagenous fibers were formed between the pleural surface and the external elastic membrane $(\times 25)$.

and pleural indentation at the tumor site (Figure 2B). A thoracoscopic right upper lobectomy was performed, and the CLE probe was brought into contact with the visceral pleura of the resected lung for observation. A regular pattern of network structure was confirmed at the site of the normal pleura away from the tumor (Figure 2C). Autofluorescence-positive structures could not be identified in the central part of the pleural change (Figure 2D). The pleura with VPI was depressed, and collagenous fiber proliferation was observed on the surface layer (Figure $2 E$ ).

Based on these findings, an autofluorescence-positive network structure can be observed in the normal visceral pleura; however, we found that when the presence of a tumor causes changes such as depressions in the visceral pleura, further changes are confirmed in autofluorescence findings. This study was designed based on the hypothesis that these autofluorescence-positive structures may disappear when the visceral pleura has been pathologically invaded by cancer.

\section{Pathological examination}

A pathological examination was performed to identify the autofluorescence-positive structures in the visceral pleura observed by CLE. Normal lung, lung cancer tissue without VPI, and lung cancer tissue with VPI were included in this examination. The abovementioned tissue was placed in a cassette to prepare a formalin-fixed paraffin-embedded block so that a section horizontal to the visceral pleural surface could be prepared. This block was sliced to a thickness of $10 \mu \mathrm{m}$, and when the surface of the visceral pleural surface was exposed, the sections were placed on a glass slide and stained with EVG.

\section{Setting the diagnostic criteria for VPI of lung cancer by CLE}

Lung resection was performed on 35 eligible cases, and CLE observations and video recordings were performed on the 
Table 1 Patient characteristics

\begin{tabular}{|c|c|c|}
\hline Factor & Ex vivo study $(\mathrm{n}=35)$ & In vivo study $(\mathrm{n}=15)$ \\
\hline \multicolumn{3}{|l|}{ Age } \\
\hline Sex & 71.5 (50 to 85$)$ & 70.6 (49 to 84 ) \\
\hline Male & 21 & 10 \\
\hline Female & 14 & 5 \\
\hline \multicolumn{3}{|c|}{ Histological type } \\
\hline Ad & 25 & 12 \\
\hline $\mathrm{Sq}$ & 9 & 3 \\
\hline Small & 1 & \\
\hline Total size $(\mathrm{cm})$ & $2.1(0.9-3.8)$ & $2.3(0.4-4.6)$ \\
\hline Solid size $(\mathrm{cm})$ & $1.4(0-3.7)$ & $1.9(0-4.6)$ \\
\hline SUV max & $5.3(0.5-28.3)$ & $5.1(0.7-11.1)$ \\
\hline \multicolumn{3}{|l|}{ Pathological VPI } \\
\hline $\mathrm{pl0}$ & 29 & 12 \\
\hline pl1 & 6 & 3 \\
\hline
\end{tabular}

Ad, adenocarcinoma; Sq, squamous cell carcinoma; Small, small cell carcinoma; SUV max, maximum standardized uptake value; VPI, visceral pleural invasion.

resected lungs (ex vivo study). Regarding patient background, the average age was 70.1 years (range, 50-85 years) with 21 males and 14 females. Histological types were 25 cases of adenocarcinoma, 9 cases of squamous cell carcinoma, and 1 case of small cell carcinoma. The average total tumor size on CT findings was $2.1 \mathrm{~cm}(0.9-3.8 \mathrm{~cm})$, and the average solid component size was $1.4 \mathrm{~cm}(0-3.7 \mathrm{~cm})$. First, the CLE probe was brought into contact with the normal part without a tumor, confirming the presence of an autofluorescence-positive network structure. Following this, the CLE probe was brought into contact with the part with the strongest pleural change, the part where the autofluorescence-positive structure disappeared most was identified, and a 10-second video was recorded at that part. Pathological evaluation of visceral pleural infiltration was performed by EVG staining in 6 cases with VPI (pl1) and 29 cases without VPI (pl0) (Table 1).

Three thoracic surgeons blinded to clinical information including CT findings and pathologic diagnosis of cases viewed the videos and independently determined the defect ratio of autofluorescence-positive structures based on a scale of five $(0 \%, 25 \%, 50 \%, 75 \%, 100 \%)$. Based on this result and the pathological VPI diagnosis, an ROC curve was created and set as the diagnostic criteria for VPI of lung cancer by CLE. The curve shows the optimal percentage for the disappearance range of the autofluorescence-positive structure.

\section{Diagnosis of VPI by CT findings and intraoperative observation under white light}

Three thoracic surgeons who participated in a study of VPI diagnosis by CLE evaluated VPI of the same 35 patients by CT findings and intraoperative observation under white light. For VPI diagnosis by CT findings, the surgeons independently reported that VPI was positive when the tumor showed the following CT findings: pleural contact, pleural thickening, solid proportion $>50 \%$, and lesion size $>20 \mathrm{~mm}$. For VPI diagnosis by intraoperative observation under white light, the surgeons were blind to the CT findings of the cases. A 10-s video of each case was played to provide the surgeons the best view showing the pleural surface where the tumor was located, inducing morphological change. The surgeons revealed that VPI is positive when the pleura showed the following CT findings: whitish change, granular change, and hypervascularization of the pleural surface.

\section{Validation study for diagnosis of lung cancer VPI}

A validation study of VPI diagnosis by CLE was performed on 15 eligible cases (in vivo study). Surgery was performed using three-port thoracoscopic surgery. After observing the thoracic cavity, the CLE probe progressed into the thoracic cavity from the ventral port. The tip of the probe was guided to the site of pleural change (Figure 3). The visceral pleura was observed by CLE, and based on the results of the above examination, the presence of VPI was diagnosed by CLE if a $50 \%$ or higher defect ratio was noted in the autofluorescence-positive structure. Evaluation of pathological VPI after lung resection was performed using EVG staining.

\section{Statistical analysis}

Statistical analyses were performed using IBM SPSS Statistics (version 22; IBM Corp., Armonk, NY, USA). The sensitivity, specificity, positive predictive value, negative predictive value, and accuracy for the three CLE imaging cutoffs were calculated. Receiver operating characteristic (ROC) curve analysis was conducted to evaluate the 


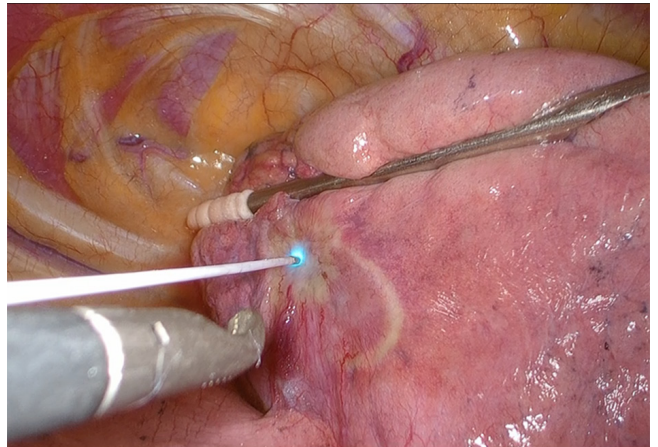

Figure 3 The CLE probe progressed into the thoracic cavity and the tip of the probe was guided to the site of pleural change.

diagnostic performance of CLE. A weighted $\kappa$ coefficient was used to assess the defect ratio of autofluorescencepositive structure agreement, and Cohen's $\kappa$ was also used to evaluate inter-reader agreement at two cutoffs. The $\kappa$ values were interpreted as follows: $0.00-0.20$, poor agreement; $0.21-0.40$, fair agreement; $0.41-0.60$, moderate agreement; $0.61-0.80$, substantial agreement; and $0.81-1.00$, almost perfect agreement. The threshold for statistical significance was set at $\mathrm{P}<0.05$.

\section{Results}

\section{Pathological examination}

In a normal lung, CLE findings of the visceral pleura showed a regular pattern of autofluorescence-positive network (Figure 4A), and in lung cancer tissue without VPI, coarsening and cord-like changes in the network were observed (Figure 4B). In lung cancer tissue with VPI, the network structure disappeared (Figure 4C). An examination was performed using the same specimens observed by CLE. The EVG-stained specimen findings of the normal lung showed that the pattern of elastic fibers was similar to the abovementioned network structure and that the CLE findings and the pattern of elastic fibers were similar in each specimen with and without VPI (Figure 4D-4F). Based on these results, we consider that the structure observed by CLE was the external elastic membrane of the visceral pleura.

\section{Setting the diagnostic criteria for VPI of lung cancer by CLE}

The sensitivity, specificity, positive and negative predictive values, and accuracy for VPI using defect ratios of autofluorescence-positive structure cutoff of $\geq 50 \%$ were $83.3-100.0 \%, 57.7-73.1 \%, 35.3-41.7 \%, 95.0-100.0 \%$, and $75.0-78.1 \%$, respectively. The corresponding values using the defect ratio of autofluorescence-positive structure cutoff of $\geq 75 \%$ were $66.7-66.7 \%, 84.6-92.3 \%, 50.0-66.7 \%$, 91.7-92.0\%, and $84.4-87.5 \%$, respectively. In contrast, a cutoff of $\geq 25 \%$ demonstrated low specificity, positive predictive values, and accuracy. A cutoff of $100 \%$ demonstrated low sensitivity (Table 2). The AUCs for the defect ratio of the autofluorescence-positive structure were 0.91 for reader 1 and 0.86 for reader 2 and 0.89 for reader 3 (Figure 5).

\section{Inter-reader agreement in the determination of defect ratio of autofluorescence-positive structure}

The weighted $\kappa$ values for agreement in the defect ratio of autofluorescence-positive structures between readers 1 and 2, 2 and 3, and 1 and 3 were 0.68 (95\% CI, 0.58-0.77), 0.75 (95\% CI, 0.67-0.84), and 0.71 (95\% CI, 0.63-0.81), respectively, which indicated substantial agreement (Table 3).

\section{Diagnosis of VPI by CT findings and intraoperative observation under white light}

The sensitivity, specificity, positive and negative predictive values, and accuracy for VPI using CT findings were $83.3 \%, 62.1-75.9 \%, 31.3-41.7 \%, 94.7-95.7 \%$, and $65.7-77.1 \%$, respectively. The sensitivity, specificity, positive and negative predictive values, and accuracy for VPI of intraoperative observation under white light were $66.7-83.3 \%, 69.0-72.4 \%, 30.8-38.5 \%, 90.9-95.5 \%$, and 68.6-74.3\%, respectively (Table 4).

\section{Validation study for diagnosis of VPI of lung cancer}

In terms of patient background, of the 15 eligible cases in the prospective validation study, the average age was 70.6 years (range, 49-84 years) with 10 males and 5 females. The histological types were 12 cases of adenocarcinoma and 3 cases of squamous cell carcinoma. The average total tumor size was $2.3 \mathrm{~cm}(0.4-4.6 \mathrm{~cm})$ and the average solid component size was $1.9 \mathrm{~cm}(0-4.6 \mathrm{~cm})$. There were 12 cases without pathologic VPI (pl0) and 3 cases with VPI (pl1) (Table 1). VPI was diagnosed if the defect ratio of the autofluorescence-positive structure was $50 \%$ or higher. the CLE probe progressed into the thoracic cavity from the 

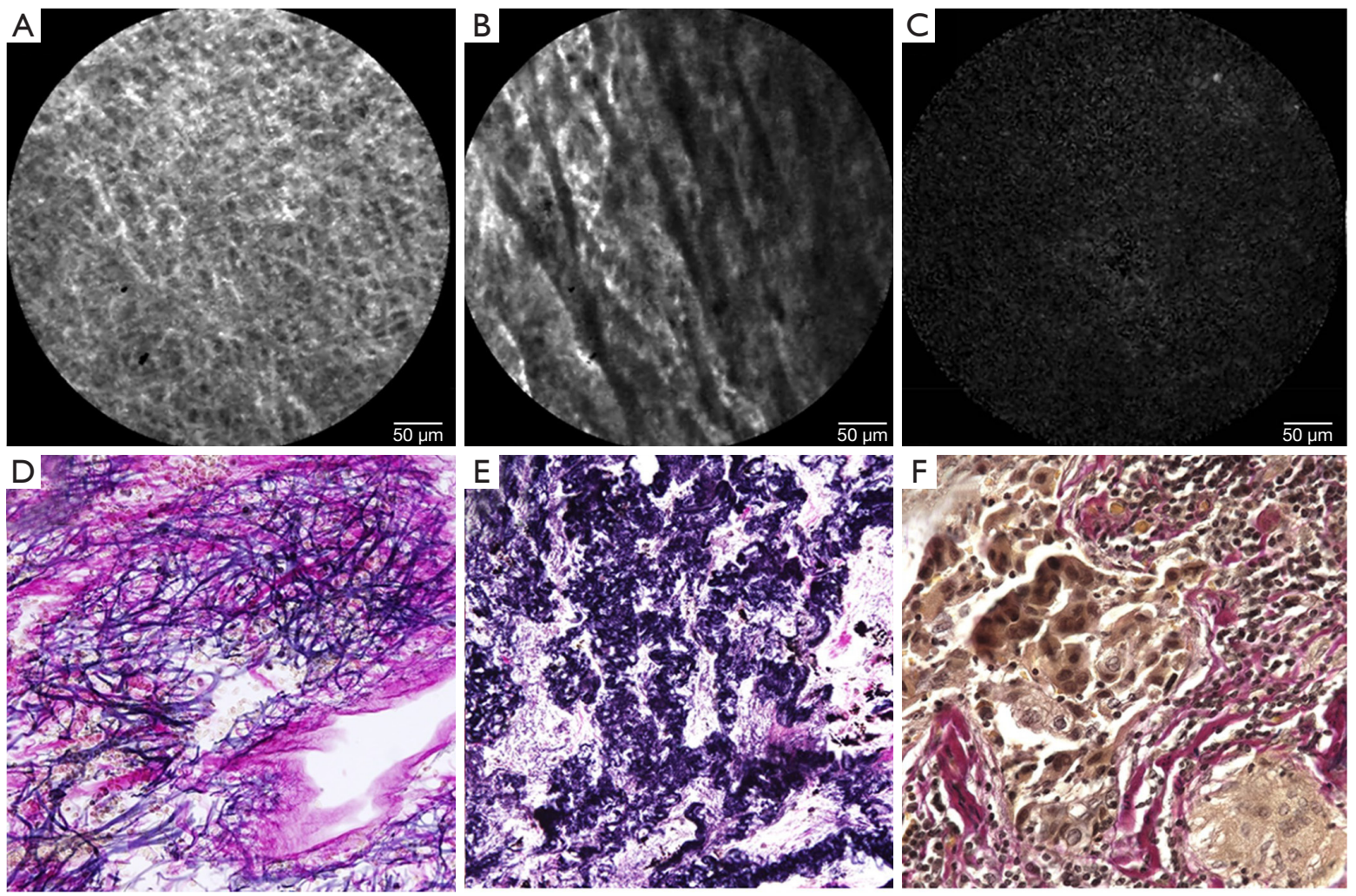

Figure 4 CLE findings and Elastica van Gieson stain in the horizontal section of the visceral pleura. (A) CLE findings of the visceral the pleura in normal lung shows a network structure; (B) CLE findings of the visceral pleura at the tumor site in non-VPI cases show thick and wavy structures; (C) autofluorescence-positive structures were not noted. CLE findings of the visceral pleura at the tumor site in cases with VPI; (D) Elastica van Gieson stain in the horizontal section of the visceral pleura in (A) shows elastic fibers with a network structure ( $\times 600)$; (E) Elastica van Gieson stain in the horizontal section of the visceral pleura in (B) shows thick and wavy elastic fibers ( $\times 600)$; (F) Elastica van Gieson stain in the horizontal section of the visceral pleura in (C) shows an increase in collagenous fibers with tumor cells $(\times 600)$.

ventral port. The tip of the probe was guided to the site of pleural change. It took about 5 minutes to observe the pleural surface by CLE and diagnose VPI. No adverse event was encountered during the validation study. As a result, sensitivity was $100 \%$, specificity was $83.3 \%$, and diagnostic accuracy rate was $86.7 \%$.

\section{Discussion}

VPI is a significant prognostic factor for lung cancer, and the prognosis for patients with VPI is poor even for small lung cancers of $2 \mathrm{~cm}$ or smaller $(6,7)$. In addition, it has been reported that the frequency of lymph node metastasis is high in patients with VPI (6). Based on these facts, it is suggested that sublobar resection should not be performed for cases with suspected VPI even for lung cancer of $2 \mathrm{~cm}$ or smaller (3). However, it is difficult to accurately diagnose VPI before and during surgery (17). Based on the general perception that VPI is pathologically diagnosed postoperatively, there has been no discussion surrounding the inclusion of VPI in the eligibility criteria for sublobar resection. VPI diagnosis by CLE is simple, non-invasive, and has a high accuracy rate; therefore, it may be useful in deciding the indication for sublobar resection. If VPI was diagnosed by CLE, the technique would be to perform lobectomy and standard lymph node dissection without performing sublobar resection, because lymph node metastases are more frequent in patients with VPI (6), which is thought to be one of the reasons of the worse prognosis.

In our ex vivo study, the sensitivity, specificity, positive and negative predictive values, and accuracy for VPI using defect ratios of autofluorescence-positive structure cutoff of $\geq 50 \%$ were $83.3-100.0 \%, 57.7-73.1 \%, 35.3-41.7 \%$, 
Table 2 Performance of CLE imaging for the diagnosis of pathologic visceral pleural invasion in lung cancer

\begin{tabular}{|c|c|c|c|c|c|}
\hline Defect ratio values & Sensitivity (\%) & Specificity (\%) & PPV (\%) & NPV (\%) & Accuracy (\%) \\
\hline Defect ratio $\geq 25 \%$ & 100.0 & 46.2 & 30.0 & 100.0 & 56.3 \\
\hline Defect ratio $\geq 50 \%$ & 83.3 & 73.1 & 41.7 & 95.0 & 75.0 \\
\hline Defect ratio $\geq 75 \%$ & 66.7 & 88.5 & 57.1 & 92.0 & 84.4 \\
\hline \multicolumn{6}{|l|}{ Reader 2} \\
\hline Defect ratio $\geq 25 \%$ & 100.0 & 38.5 & 27.3 & 100.0 & 56.3 \\
\hline Defect ratio $\geq 50 \%$ & 100.0 & 57.7 & 35.3 & 100.0 & 75.0 \\
\hline Defect ratio $\geq 75 \%$ & 66.7 & 84.6 & 50.0 & 91.7 & 84.4 \\
\hline Defect ratio $\geq 25 \%$ & 100.0 & 38.5 & 27.3 & 100.0 & 50.0 \\
\hline Defect ratio $\geq 50 \%$ & 100.0 & 73.1 & 46.2 & 100.0 & 78.1 \\
\hline Defect ratio $\geq 75 \%$ & 66.7 & 92.3 & 66.7 & 91.7 & 87.5 \\
\hline Defect ratio $=100 \%$ & 16.7 & 92.3 & 33.3 & 82.8 & 78.1 \\
\hline
\end{tabular}

CLE, confocal laser endomicroscopy; PPV, positive predictive value; NPV, negative predictive value.

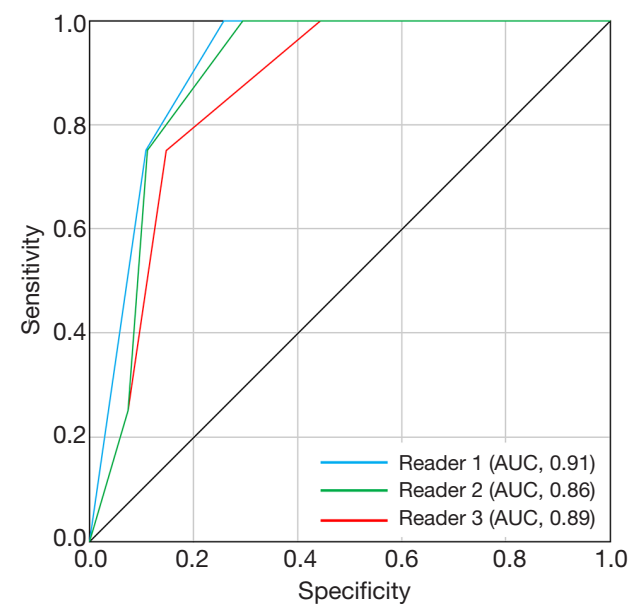

Figure 5 Receiver operating characteristic curve analysis of pathological visceral pleural invasion using defect ratio of autofluorescence-positive structure assigned by three readers.

95.0-100.0\%, and 75.0-78.1\%, respectively. Furthermore, in the validation study, the sensitivity, specificity, and accuracy were $100 \%, 83.3 \%$, and $86.7 \%$, respectively. There have been several studies about preoperative VPI diagnosis using CT findings. The sensitivity, specificity, positive and negative predictive values, and accuracy for VPI using CT findings were $36.4-45.0 \%, 91.0-92.9 \%, 76.2-94.0 \%$, $36.0-69.6 \%$, and $57.0-71.0 \%$, respectively $(18,19)$. Tanaka et al. reported fluorine-18-fluorodeoxyglucose uptake for risk stratification of VPI of lung adenocarcinoma, and the sensitivity, specificity, positive and negative predictive values, and accuracy for VPI using maximum standardized uptake value were $63.6 \%, 88.1 \%, 51.9 \%, 92.3 \%$, and $84.0 \%$, respectively (20). As regards intraoperative VPI diagnosis, the sensitivity, specificity, and accuracy using an autofluorescence endoscopy system were $83.3 \%, 73.7 \%$, and $76.0 \%$, respectively (17). Kitada et al. reported an autofluorescence observation method after oral intake of 5-amino-levulinic acid with the sensitivity, specificity, positive predictive value, and negative predictive value of $100 \%, 58.0 \%, 63.1 \%$, and $100 \%$, respectively (21).

It has been reported that the use of CLE for bronchoscopy allows differentiation between neoplastic and nonneoplastic lung tissues (15). However, no study has used CLE for intraoperative VPI diagnosis in lung cancer surgery. By comparing with the findings of EVG staining, the autofluorescence-positive network structure of the visceral pleura observed by CLE was shown to be elastic fibers in the external elastic membrane. Based on the electron 
Table 3 Interobserver agreement for the defect ratio of autofluorescence-positive structures

\begin{tabular}{lccc}
\hline \multirow{2}{*}{ Defect ratio values } & \multicolumn{3}{c}{$\kappa$ coefficient (95\% Cl) } \\
\cline { 2 - 4 } & Readers A and B & Readers A and C & Readers B and C \\
\hline Overall defect ratios & $0.68(0.58-0.77)$ & $0.75(0.67-0.84)$ & $0.71(0.63-0.81)$ \\
Defect ratio $\geq 50 \%$ & $0.69(0.57-0.81)$ & $0.80(0.70-0.91)$ & $0.75(0.64-0.86)$ \\
Defect ratio $\geq 75 \%$ & $0.91(0.83-0.98)$ & $0.71(0.66-0.86)$ & $0.63(0.57-0.79)$ \\
\hline
\end{tabular}

Table 4 Performance of CLE, CT, and white light for the diagnosis of pathologic visceral pleural invasion in lung cancer

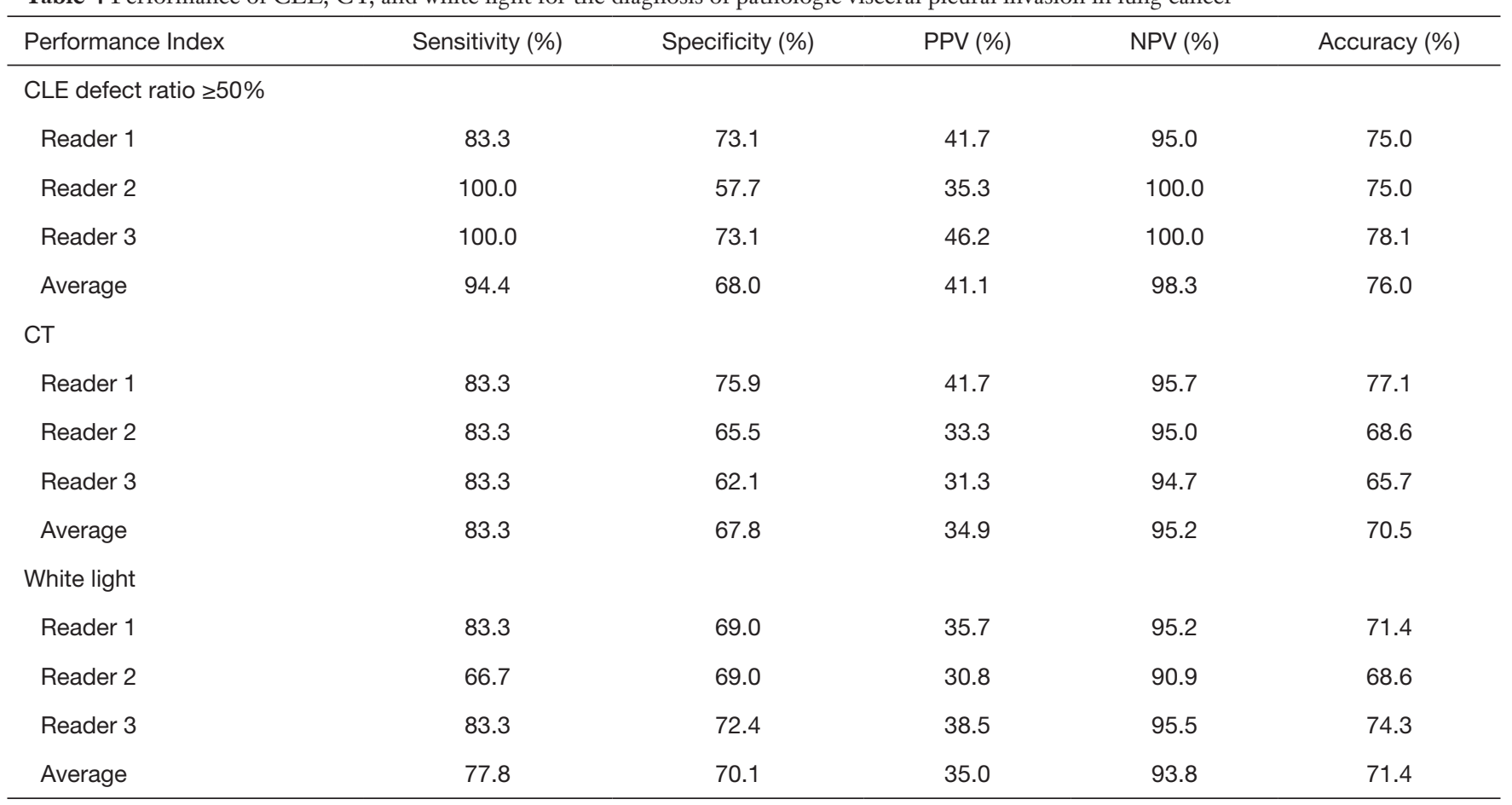

CLE, confocal laser endomicroscopy; CT, computed tomography; PPV, positive predictive value; NPV, negative predictive value.

microscopic findings of the visceral pleura, it is known that the structure of the visceral pleura is composed of 2-3 layers of elastin fibers, forming a regular structure (22). Elastin is a substance that emits autofluorescence, and its excitation wavelength peaks at approximately $405 \mathrm{~nm}$ (11). Since the Cellvizio system used for the observation of the visceral pleura emits excitation light peaking at $488 \mathrm{~nm}$, we believe that the network structure observed in the visceral pleura is the elastin fiber of the external elastic membrane. Studies applying CLE to bronchoscopy have been reported, and it has been shown that a network of elastin fibers in the alveolar structure can be observed by CLE (11).

In previous studies, we reported that the use of an autofluorescence endoscopy system improved the diagnostic accuracy rate of VPI (17). VPI is deemed present when the green autofluorescence light is diminished or disappears. When intraoperative VPI diagnosis was performed, the sensitivity, specificity, and correct diagnosis rate in white light were $64.6 \%, 53.9 \%$, and $56.5 \%$, respectively. In autofluorescence observation, these improved to $83.3 \%$, $73.7 \%$, and $76.0 \%$, respectively. The excitation light in the autofluorescence mode of the D-Light AutoFluorescence system (Karl Storz, Tuttlingen, Germany) used in this study was $375-440 \mathrm{~nm}$, which is close to that of the Cellvizio system. Particularly, this system visualized the external elastic membrane of the visceral pleura, and we believe that the autofluorescence change was related to the invasion of cancer cells into the visceral pleura. Additionally, the possibility of 
distinguishing between normal and lung diseases by analyzing the thickness of elastin has been reported (23).

There are two possible reasons for the disappearance of autofluorescence-positive structures in areas with VPI. The first is the partial disappearance caused by the destruction of the external elastic membrane associated with cancer invasion. CLE visualizes elastin fibers but not cancer cells; the autofluorescence-positive structure in the invasion part appears to be missing. Another reason is that pleural thickening occurs on the surface of the pleura rather than the external elastic membrane. Since the observation depth of the CLE probe used in this study was 0-50 $\mu \mathrm{m}$ (15), if autofluorescence-negative tissue grows on the visceral pleural surface and exceeds this thickness, the external elastic membrane cannot be observed. It is known that the visceral pleural elastic layer could be invaded in three patterns: (I) without eliciting any secondary changes, (II) with prominent elastic reduplication and inflammatory infiltrates, and (III) with thick fibroblastic proliferation (24). That is, if inflammatory infiltrates or collagenous fiber proliferation are present just below the pleural surface, the autofluorescence of the external elastic membrane disappears. In our study, we confirmed the abovementioned histological findings in cases with VPI. Though the sensitivity of CLE observation in diagnosing VPI was high, the specificity was relatively low. The reason why there were few false-positive cases was that the autofluorescencepositive network structure was hardly observed at the visceral pleura that showed anthracosis or pleural thickening caused by obstructive pneumonia.

The limitation of this study was its small number of cases from a single institution. Therefore, to apply this method clinically, performing a multicenter clinical research with a large number of cases is required. Moreover, the study included NSCLC patients with a relatively large tumor although intraoperative CLE diagnosis of VPI was expected to be applied to the indication of sublobar resection for small-sized peripheral NSCLC. In addition, the VPI diagnosis by the Cellvizio system is subjective to the evaluator, which may result in poor reproducibility.

\section{Conclusions}

In conclusion, VPI diagnosis by the Cellvizio system is simple, non-invasive, and has a high accuracy rate; thus, it may be useful in deciding the indication for reductive surgery. Furthermore, verification of diagnostic accuracy for a large number of cases is warranted.

\section{Acknowledgments}

Funding: This work was supported by a Grant-in-Aid for Young Scientists (B) No. 17K16611 from the Ministry of Education, Culture, Sports, Science and Technology (MEXT) from 2017 to 2019.

\section{Footnote}

Reporting checklist: The authors have completed the STARD reporting checklist. Available at https://dx.doi. org/10.21037/jtd-21-137

Data Sharing Statement: Available at https://dx.doi. org/10.21037/jtd-21-137

Peer Review File: Available at https://dx.doi.org/10.21037/ jtd-21-137

Conflicts of Interest: All authors have completed the ICMJE uniform disclosure form (available at https://dx.doi. org/10.21037/jtd-21-137). KK serves as an unpaid editorial board member of Journal of Thoracic Disease. HT serves as an unpaid editorial board member of Fournal of Thoracic Disease from Feb 2021 to Jan 2023. The other authors have no conflicts of interest to declare.

Ethical Statement: The authors are accountable for all aspects of the work in ensuring that questions related to the accuracy or integrity of any part of the work are appropriately investigated and resolved. The study was conducted in accordance with the Declaration of Helsinki (as revised in 2013). The study was approved by the Ethics Review Board of the Tokushima University Hospital (approval number 3362), and informed consent was obtained from all the participants.

Open Access Statement: This is an Open Access article distributed in accordance with the Creative Commons Attribution-NonCommercial-NoDerivs 4.0 International License (CC BY-NC-ND 4.0), which permits the noncommercial replication and distribution of the article with the strict proviso that no changes or edits are made and the original work is properly cited (including links to both the formal publication through the relevant DOI and the license). See: https://creativecommons.org/licenses/by-nc-nd/4.0/.

\section{References}

1. Global Burden of Disease Cancer Collaboration; 
Fitzmaurice C, Dicker D, et al. The Global Burden of Cancer 2013. JAMA Oncol 2015;1:505-27.

2. Goldstraw P, Chansky K, Crowley J, et al. The IASLC Lung Cancer Staging Project: Proposals for Revision of the TNM Stage Groupings in the Forthcoming (Eighth) Edition of the TNM Classification for Lung Cancer. J Thorac Oncol 2016;11:39-51.

3. Okada M, Koike T, Higashiyama M, et al. Radical sublobar resection for small-sized non-small cell lung cancer: a multicenter study. J Thorac Cardiovasc Surg 2006;132:769-75.

4. Yoshikawa K, Tsubota N, Kodama K, et al. Prospective study of extended segmentectomy for small lung tumors: the final report. Ann Thorac Surg 2002;73:1055-8; discussion 1058-9.

5. Nakamura K, Saji H, Nakajima R, et al. A phase III randomized trial of lobectomy versus limited resection for small-sized peripheral non-small cell lung cancer (JCOG0802/ WJOG4607L). Jpn J Clin Oncol 2010;40:271-4.

6. Yoshida J, Nagai K, Asamura H, et al. Visceral pleura invasion impact on non-small cell lung cancer patient survival: its implications for the forthcoming TNM staging based on a largescale nation-wide database. J Thorac Oncol 2009;4:959-63.

7. Lakha S, Gomez JE, Flores RM, et al. Prognostic significance of visceral pleural involvement in early-stage lung cancer. Chest 2014;146:1619-26.

8. Inoue $\mathrm{M}$, Minami $\mathrm{M}$, Shiono $\mathrm{H}$, et al. Clinicopathologic study of resected, peripheral, small-sized, non-small cell lung cancer tumors of $2 \mathrm{~cm}$ or less in diameter: pleural invasion and increase of serum carcinoem-bryonic antigen level as predictors of nodal involvement. J Thorac Cardiovasc Surg 2006;131:988-93.

9. Kawase A, Yoshida J, Miyaoka E, et al. Visceral pleural invasion classification in non-small-cell lung cancer in the 7 th edition of the tumor, node, metastasis classification for lung cancer: validation analysis based on a large-scale nationwide database. J Thorac Oncol 2013;8:606-11.

10. Shimada Y, Yoshida J, Hishida T, et al. Predictive factors of pathologically proven noninvasive tumor characteristics in T1aN0M0 peripheral non-small cell lung cancer. Chest 2012;141:1003-9.

11. Thiberville L, Salaün M, Lachkar S, et al. Human in vivo fluorescence microimaging of the alveolar ducts and sacs during bronchoscopy. Eur Respir J 2009;33:974-85.

12. Kiesslich R, Burg J, Vieth M, et al. Confocal laser endoscopy for diagnosing intraepithelial neoplasias and colorectal cancer in vivo. Gastroenterology 2004;127:706-13.

13. Rasmussen DN, Karstensen JG, Riis LB, et al. Confocal Laser Endomicroscopy in Inflammatory Bowel Disease--A
Systematic Review. J Crohns Colitis 2015;9:1152-9.

14. Thong PS, Olivo M, Kho KW, et al. Laser confocal endomicroscopy as a novel technique for fluorescence diagnostic imaging of the oral cavity. J Biomed Opt 2007;12:014007.

15. Fuchs FS, Zirlik S, Hildner K, et al. Confocal laser endomicroscopy for diagnosing lung cancer in vivo. Eur Respir J 2013;41:1401-8.

16. Thiberville L, Moreno-Swirc S, Vercauteren T, et al. In vivo imaging of the bronchial wall microstructure using fibered confocal fluorescence microscopy. Am J Respir Crit Care Med 2007;175:22-31.

17. Takizawa H, Kondo K, Kawakita N, et al. Autofluorescence for the diagnosis of visceral pleural invasion in non-smallcell lung cancer. Eur J Cardiothorac Surg 2018;53:987-92.

18. Hsu JS, Jaw TS, Yang CJ, et al. Convex border of peripheral non-small cell lung cancer on CT images as a potential indicator of pleural invasion. Medicine (Baltimore) 2017;96:e7323.

19. Hsu JS, Han IT, Tsai TH, et al. Pleural Tags on CT Scans to Predict Visceral Pleural Invasion of Non-Small Cell Lung Cancer That Does Not Abut the Pleura. Radiology 2016;279:590-6.

20. Tanaka T, Shinya T, Sato S, et al. Predicting pleural invasion using HRCT and 18F-FDG PET/CT in lung adenocarcinoma with pleural contact. Ann Nucl Med 2015;29:757-65.

21. Kitada M, Ohsaki Y, Yasuda S, et al. Photodynamic diagnosis of visceral pleural invasion of lung cancer with a combination of 5-aminolevulinic acid and autofluorescence observation systems. Photodiagnosis Photodyn Ther 2017;20:10-5.

22. Toshima M, Ohtani Y, Ohtani O. Three-dimensional architecture of elastin and collagen fiber networks in the human and rat lung. Arch Histol Cytol 2004;67:31-40.

23. Bondesson D, Schneider MJ, Silbernagel E, et al. Automated evaluation of probe-based confocal laser endomicroscopy in the lung. PLoS One 2020;15:e0232847.

24. Gallagher B, Urbanski SJ. The significance of pleural elastica invasion by lung carcinomas. Hum Pathol 1990;21:512-7.

Cite this article as: Sawada T, Takizawa H, Aoyama M, Kawakita N, Miyamoto N, Sakamoto S, Takashima M, Matsumoto D, Toba H, Kawakami Y, Yoshida M, Kondo K, Tangoku A. Diagnosis of visceral pleural invasion using confocal laser endomicroscopy during lung cancer surgery. J Thorac Dis 2021;13(8):4742-4752. doi: 10.21037/jtd-21-137 